This is an Open Access article, distributed under the terms of the Creative Commons Attribution licence (http://creativecommons.org/licenses/by/4.0/), which permits unrestricted re-use, distribution, and reproduction in any medium, provided the original work is properly cited.

doi: $10.1017 /$ jfm.2017.413

\title{
Extension of Kelvin's minimum energy theorem to incompressible fluid domains with open regions
}

\author{
Tony Saad $^{1}$ and Joseph Majdalani ${ }^{2}{ }^{\dagger}$ \\ ${ }^{1}$ Department of Chemical Engineering, University of Utah, Salt Lake City, UT 84102, USA \\ ${ }^{2}$ Department of Aerospace Engineering Auburn University, Auburn, AL 36849, USA
}

(Received 24 March 2017; revised 8 June 2017; accepted 8 June 2017;

first published online 20 July 2017)

\begin{abstract}
Kelvin's minimum energy theorem predicts that the irrotational motion of a homogeneously incompressible fluid in a simply connected region will carry less kinetic energy than any other profile that shares the same normal velocity conditions on the domain's boundary. In this work, Kelvin's analysis is extended to regions with boundaries on which the normal velocity requirements are relaxed. Given the ubiquity of practical configurations in which such boundaries exist, the question of whether Kelvin's theorem continues to hold is one of significant interest. In reconstructing Kelvin's proof, we find it useful to define a net rotational velocity as the difference between the generally rotational flow and the corresponding potential motion. In Kelvin's classic theorem, the normal component of the net rotational velocity at all domain boundaries is zero. In contrast, the present analysis derives a sufficient condition for ensuring the validity of Kelvin's theorem in a domain where the normal component of net rotational velocity at some or all of the boundaries is not zero. The corresponding criterion requires the evaluation of a simple surface integral over the boundary.
\end{abstract}

Key words: general fluid mechanics, mathematical foundations, Navier-Stokes equations

\section{Introduction}

Kelvin's theorems occupy a central role in understanding the motion of ideal fluids as they help to establish basic connections between purely irrotational velocity potentials and rotational fields. Being relevant to both classical and quantum fluids, they continue to receive attention in various fundamental studies such as those pertaining to turbulence (Chen et al. 2006; Gregory 2006) and aerodynamic lift (Wu, Ma \& Zhou 2006). Other interesting applications include a generalization of the minimum energy theorem to equivorticity flows (Arnold 1965) and the use of variational theory to specify the conditions for minimizing the kinetic energy of vortex

$\dagger$ Email address for correspondence: joe.majdalani@auburn.edu 
motions (Chefranov \& Chefranov 2003). Of the many rich contributions attributed to Kelvin, the present work focuses on the minimum energy theorem. Devised in 1849 , this theorem states that the irrotational motion $\overline{\boldsymbol{u}}$ of an incompressible fluid in a simply connected region contains less kinetic energy than any other motion $\boldsymbol{u}$ with the same normal velocity at its boundary, $\overline{\boldsymbol{u}} \cdot \boldsymbol{n}=\boldsymbol{u} \cdot \boldsymbol{n}$ (Thomson 1849). The additional caveat is that, for a fluid extending to infinity, the theorem requires a vanishing normal velocity at the far-field boundary (Lamb 1895; Batchelor 1967).

Several direct consequences may be deduced from Kelvin's theorem. First, it precludes the onset of irrotational motion in a simply connected, non-deformable region with rigid walls, where the potential velocity is nullified at all points on the boundary. Such a scenario is consistent with a system at rest or one with no kinetic energy. Second, for a non-deformable region with fixed rigid walls, no irrotational motion may be sustained when the velocity at infinity vanishes. Third, when the velocity at infinity is either null or uniform, a unique irrotational solution may be associated with a given motion of the internal boundary (Rouse 1959). In this work, we find that Kelvin's minimum energy theorem continues to hold in regions where $\overline{\boldsymbol{u}} \cdot \boldsymbol{n} \neq \boldsymbol{u} \cdot \boldsymbol{n}$, provided that a simple criterion is met.

\section{Basic analysis}

In what follows, we assume that the rotational velocity fields are incompressible and regular.

THEOREM 1 (For homogeneous and incompressible fluids with open boundaries).

The irrotational motion $\overline{\boldsymbol{u}}$ of a steady, homogeneous and incompressible fluid in a simply connected fluid region $\mathcal{V}$ contains less kinetic energy than any another motion $\boldsymbol{u}$, with or without the same normal velocity at its boundary, provided that the following sufficient condition is met

$$
T=\iint_{\mathcal{S}} \phi \tilde{\boldsymbol{u}} \cdot \boldsymbol{n} \mathrm{d} \mathcal{S} \geqslant 0,
$$

where $\tilde{\boldsymbol{u}}=\boldsymbol{u}-\overline{\boldsymbol{u}}$ defines the net rotational component of the motion and $\phi, \boldsymbol{n}$, and $\mathcal{S}$ denote the velocity potential, normal unit vector and boundary surface, respectively.

Proof. With $\overline{\boldsymbol{u}}=\nabla \phi$ being a steady, single-valued velocity component of a homogeneously incompressible fluid occupying a simply connected volume of fluid $\mathcal{V}$, then $\tilde{\boldsymbol{u}}=\boldsymbol{u}-\overline{\boldsymbol{u}}$ refers to the (net) rotational contribution and difference between the velocity of another (rotational) motion satisfying continuity and the potential solution $\overline{\boldsymbol{u}}$ (see figure 1). These fields are incompressible and so, by virtue of mass conservation, one may put

$$
\nabla \cdot \overline{\boldsymbol{u}}=\nabla \cdot \boldsymbol{u}=\nabla \cdot \tilde{\boldsymbol{u}}=0 .
$$

Pursuant to Kelvin's argument, $\boldsymbol{u}$ and $\overline{\boldsymbol{u}}$ must exhibit the same normal velocity along the boundary of $\mathcal{V}$ or else vanish. For steady, homogeneous, incompressible motion, we choose $T$ and $\bar{T}$ to represent the specific kinetic energies associated with $\boldsymbol{u}$ and $\overline{\boldsymbol{u}}$, respectively. Subsequently, the energy contribution due to rotationality may be 


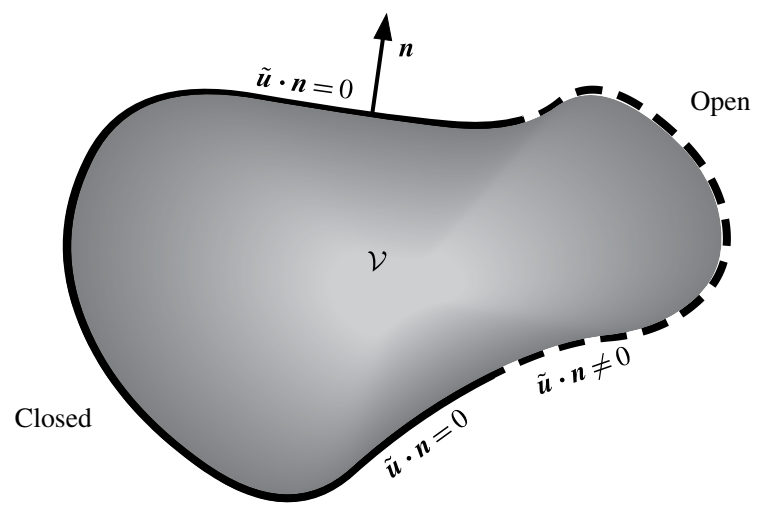

FIGURE 1. Volume of fluid showing surfaces with different net rotational velocities at the boundaries.

calculated from

$$
\begin{aligned}
\Delta T & =T-\bar{T}=\frac{1}{2} \iiint_{\mathcal{V}}\left(\boldsymbol{u}^{2}-\overline{\boldsymbol{u}}^{2}\right) \mathrm{d} \mathcal{V} \\
& =\frac{1}{2} \iiint_{\mathcal{V}}\left[(\boldsymbol{u}-\overline{\boldsymbol{u}})^{2}+2(\boldsymbol{u}-\overline{\boldsymbol{u}}) \cdot \overline{\boldsymbol{u}}\right] \mathrm{d} \mathcal{V} \\
& =\frac{1}{2} \iiint_{\mathcal{V}} \tilde{\boldsymbol{u}}^{2} \mathrm{~d} \mathcal{V}+\iiint_{\mathcal{V}} \tilde{\boldsymbol{u}} \cdot \nabla \phi \mathrm{d} \mathcal{V}
\end{aligned}
$$

The last member of (2.3) may be simplified through the use of $\tilde{\boldsymbol{u}} \cdot \nabla \phi=\nabla \cdot(\phi \tilde{\boldsymbol{u}})-$ $\phi \nabla \cdot \tilde{\boldsymbol{u}}=\boldsymbol{\nabla} \cdot(\phi \tilde{\boldsymbol{u}})$ in conjunction with the divergence theorem. One is left with

$$
\left.\begin{array}{rl}
\widetilde{T} & \equiv \frac{1}{2} \iiint_{\mathcal{V}} \tilde{\boldsymbol{u}}^{2} \mathrm{~d} \mathcal{V} \\
T_{\mathcal{S}} & \equiv \iint_{\mathcal{S}} \phi \tilde{\boldsymbol{u}} \cdot \boldsymbol{n} \mathrm{d} \mathcal{S} .
\end{array}\right\}
$$

In constructing Kelvin's theorem, the net rotational motion vanishes on all boundaries, thus yielding $\tilde{\boldsymbol{u}} \cdot \boldsymbol{n}=0$ on $\mathcal{S}$. This permits setting $T_{\mathcal{S}}=0$ in (2.4) and deducing that $\Delta T \geqslant 0$ given that $\widetilde{T} \geqslant 0$ for any rotational field. It can therefore be seen that $\tilde{\boldsymbol{u}} \cdot \boldsymbol{n}=0$ ensures that the energy associated with the potential motion remains a minimum. However, in the general case of $\tilde{\boldsymbol{u}} \cdot \boldsymbol{n} \neq 0$, it is possible for $T_{\mathcal{S}} \neq 0$. Clearly, in order for $\Delta T \geqslant 0$, it is necessary and sufficient to impose

$$
\frac{1}{2} \iiint_{\mathcal{V}} \tilde{\boldsymbol{u}}^{2} \mathrm{~d} \mathcal{V}+\iint_{\mathcal{S}} \phi \tilde{\boldsymbol{u}} \cdot \boldsymbol{n} \mathrm{d} \mathcal{S} \geqslant 0 .
$$

Recognizing that the first term in (2.5) is always positive, it is sufficient to show that

$$
T_{\mathcal{S}} \equiv \iint_{\mathcal{S}} \phi \tilde{\boldsymbol{u}} \cdot \boldsymbol{n} \mathrm{d} \mathcal{S} \geqslant 0 .
$$

Kelvin's theorem may thus be extended to the flow of homogeneously incompressible fluids in regions with open boundaries when either of the two above conditions is fulfilled. 


\section{Discussion}

According to Batchelor (1967), Kelvin's theorem ceases to hold for a multi-valued potential, such as the one corresponding to the flow in multiply connected regions, unless one selects the particular irrotational motion that bears the least kinetic energy among all potential solutions. Alternatively, if one defines a velocity potential as the difference between two possible potential solutions having the same cyclic constant $\left(\phi=\phi_{0}-\phi_{1}\right)$, then the theorem can be applied because of the uniqueness of the resulting potential.

Since velocity potentials may be specified up to an additive constant $K$, the effect of $K$ on (2.6) must be examined. In this case, we replace $\phi$ by $(\phi+K)$ in (2.6) and recover

$$
T_{\mathcal{S}}=\iint_{\mathcal{S}}(\phi+K) \tilde{\boldsymbol{u}} \cdot \boldsymbol{n} \mathrm{d} \mathcal{S}=\iint_{\mathcal{S}} \phi \tilde{\boldsymbol{u}} \cdot \boldsymbol{n} \mathrm{d} \mathcal{S} \geqslant 0,
$$

where the divergence-free property of the net rotational motion is used to reduce the above integral $\left(\iint_{\mathcal{S}} \tilde{\boldsymbol{u}} \cdot \boldsymbol{n} \mathrm{d} \mathcal{S}=0\right)$. It can thus be seen that, for single-valued velocity potentials, the extended Kelvin criterion is not affected by the addition of an arbitrary constant to the potential.

From a practical standpoint, it may be useful to check whether the evaluation of the surface integral in (2.6) will be more straightforward than the volume integral $\iiint_{\mathcal{V}}\left(\boldsymbol{u}^{2}-\overline{\boldsymbol{u}}^{2}\right) \mathrm{d} \mathcal{V}$. Such a verification will require an assessment of the algebraic complexity of the integrals in question as well as the cost of computing them individually.

In terms of algebraic complexity, it can be argued that the presence of squares in the volume integral can lead to nonlinear expressions and, hence, more tedious algebraic manipulations. In contrast, it is likely for the surface integral to require less effort to evaluate.

Another benefit of evaluating (2.6) versus (2.3) is associated with the cost of numerical computation. To illustrate this point, we define the speed-up factor as the ratio of the time needed to compute the volume integral to that needed to compute its corresponding surface integral, $t_{\mathcal{V}} / t_{\mathcal{S}}$. We then calculate the speed up for three representative problems that are well known in the community. These are (i) the Poiseuille motion in a duct, (ii) the Taylor motion in a porous channel (Taylor 1956) and (iii) the Taylor-Culick motion in a porous cylinder (Culick 1966). The corresponding computations are carried out using quadrature routines provided by the NumPy library in Python within machine precision. For the reader's convenience, speed-up results are catalogued in table 1, where the speed of computing volume integrals is seen to range from approximately 20 to 30 times the speed of computing the corresponding surface integrals.

It should be noted that, when the normal velocities on the boundary for a given potential motion and for other flows are allowed to differ, our simple condition ensures that the given potential motion will still possess the least energy. It is hoped that other researchers will be interested in generating additional verifications and proofs or, perhaps, exceptions and exclusions that we may have overlooked.

\section{Conclusion}

The present analysis seeks to address the viability of Kelvin's incompressible theorem in regions where the normal velocity components of the irrotational and rotational motions are allowed to differ. Our work suggests that Kelvin's minimum energy statement is connected to the sign of an integral that strictly depends on the 


$\begin{array}{lcccc}\text { Problem } & \phi & \overline{\boldsymbol{u}} & \boldsymbol{u} & \text { Speed up } \\ \text { Poiseuille } & z / 2 & \boldsymbol{e}_{z} / 2 & \left(1-r^{2}\right) \boldsymbol{e}_{z} & 20.6 \\ \text { Taylor } & \left(x^{2}-y^{2}\right) / 2 & x \boldsymbol{e}_{x}-y \boldsymbol{e}_{y} & (\pi x / 2) \cos (\pi y / 2) \boldsymbol{e}_{x}-\sin (\pi y / 2) \boldsymbol{e}_{y} & 22.2 \\ \text { Taylor-Culick } & -r^{2} / 2+z^{2} & -r \boldsymbol{e}_{r}+2 z \boldsymbol{e}_{z} & -r^{-1} \sin \left(\pi r^{2} / 2\right) \boldsymbol{e}_{r}+\pi z \cos \left(\pi r^{2} / 2\right) \boldsymbol{e}_{z} & 29.8\end{array}$

TABLE 1. Speed advantage of numerically computing the surface integral (2.6) relative to the volume integral (2.3) for the cases of: (i) Poiseuille flow in a duct, (ii) Taylor flow in a porous channel and (iii) Taylor-Culick flow in a porous cylinder. The speed-up factor is defined as the ratio of the time needed to compute the volume integral to that needed to compute the surface integral. Depending on the coordinate system used, $\boldsymbol{e}_{x}, \boldsymbol{e}_{y}, \boldsymbol{e}_{r}$ and $\boldsymbol{e}_{z}$ refer to the unit vectors in the $x, y, r$ and $z$ directions, respectively.

rotational flux over the boundary as well as the local velocity potential. This no longer requires a vanishing or uniform velocity field at infinity for the theorem to stand. Instead, the ability of the present analysis to account for irregular velocity distributions at fluid boundaries grants Kelvin's theorem broader applicability to fluid regions with arbitrary velocity distributions in homogeneous and incompressible flow fields.

\section{Acknowledgements}

This material is based on work supported partly by the National Science Foundation, and partly by Auburn University, Department of Aerospace Engineering, through the Hugh and Loeda Francis Chair of Excellence. The authors thank Professor J.-Z. Wu of Peking University for his insightful comments regarding the limitations of Lord Kelvin's classic energy theorem.

\section{REFERENCES}

ARNOLD, V. I. 1965 Variational principle for three-dimensional steady-state flows of an ideal fluids. Z. Angew. Math. Mech. 29 (5), 1002-1008.

Batchelor, G. K. 1967 An Introduction to Fluid Dynamics. Cambridge University Press.

Chefranov, A. \& Chefranov, S. 2003 Extrema of the kinetic energy and its dissipation rate in vortex flows. Dokl. Phys. 48 (12), 696-700.

Chen, S., Eyink, G. L., WAn, M. \& XiaO, Z. 2006 Is the Kelvin theorem valid for high Reynolds number turbulence? Phys. Rev. Lett. 97 (14), 144505-144508.

Culick, F. E. C. 1966 Rotational axisymmetric mean flow and damping of acoustic waves in a solid propellant rocket. AIAA J. 4 (8), 1462-1464.

Gregory, L. E. 2006 Cascade of circulations in fluid turbulence. Phys. Rev. E 74 (6), 066302.

LAmB, H. 1895 Hydrodynamics. Cambridge University Press.

Rouse, H. 1959 Advanced Mechanics of Fluids. Wiley.

TAYlor, G. I. 1956 Fluid flow in regions bounded by porous surfaces. Proc. R. Soc. Lond. A 234 (1199), 456-475.

Thomson, W. 1849 Notes on hydrodynamics V: on the vis-viva of a liquid in motion. Camb. Dublin Math. J. 4, 90-94.

Wu, J. Z., MA, H. Y. \& Zhou, M. D. 2006 Vorticity and Vortex Dynamics. Springer. 